\title{
S2 File
}

\section{Differential item functioning (DIF) - sex}

As can be seen from Table 1, item 4 was flagged for DIF. However, the McFadden pseudo- $\mathrm{R}^{2}$ indices suggested that the DIF effect was negligible. Additionally, the change in beta parameter for model 1 and model 2 was well below $5 \%$ in all instances. Figure 1 shows the plots for item 4 and, as can be seen from the figure (bottom-right), the impact is negligible.

Table 1. DIF test based on participants' sex

\begin{tabular}{|c|c|c|c|c|c|c|c|}
\hline Item \# & M1 vs M2 & M1 vs M3 & M2 vs M3 & $\begin{array}{c}\mathbf{R}^{2} \\
\text { (M1, M2) }\end{array}$ & $\begin{array}{c}\mathbf{R}^{\mathbf{2}} \\
\text { (M1, M3) }\end{array}$ & $\begin{array}{c}\mathbf{R}^{\mathbf{2}} \\
\text { (M2, M3) }\end{array}$ & $\begin{array}{c}\Delta \boldsymbol{\beta} \\
\text { (M1, M2) }\end{array}$ \\
\hline 1 & 0.115 & 0.177 & 0.323 & 0.003 & 0.004 & 0.001 & 0.013 \\
\hline 2 & 0.035 & 0.095 & 0.608 & 0.006 & 0.006 & 0.000 & 0.022 \\
\hline 3 & 0.782 & 0.646 & 0.372 & 0.000 & 0.001 & 0.001 & 0.001 \\
\hline 4 & 0.944 & 0.002 & 0.000 & 0.000 & 0.014 & 0.014 & 0.000 \\
\hline 5 & 0.014 & 0.013 & 0.107 & 0.008 & 0.011 & 0.003 & 0.009 \\
\hline 6 & 0.096 & 0.015 & 0.017 & 0.004 & 0.012 & 0.008 & 0.001 \\
\hline 7 & 0.575 & 0.580 & 0.378 & 0.000 & 0.001 & 0.001 & 0.001 \\
\hline 8 & 0.666 & 0.649 & 0.410 & 0.000 & 0.001 & 0.001 & 0.001 \\
\hline 9 & 0.041 & 0.084 & 0.372 & 0.009 & 0.011 & 0.002 & 0.021 \\
\hline
\end{tabular}

Notes. $\mathrm{M}=$ model number chi-square test; statistical significance $<0.01 ; R^{2}=$ McFadden pseudo- $R^{2} ; \Delta \beta=$ change in beta for model 1 and model 2.

Item True Score Functions - Item 4

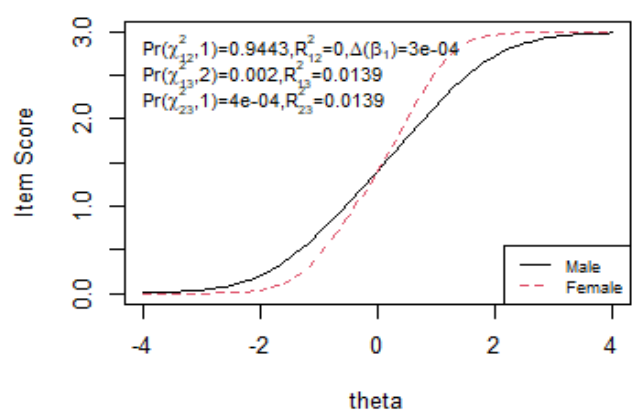

Item Response Functions

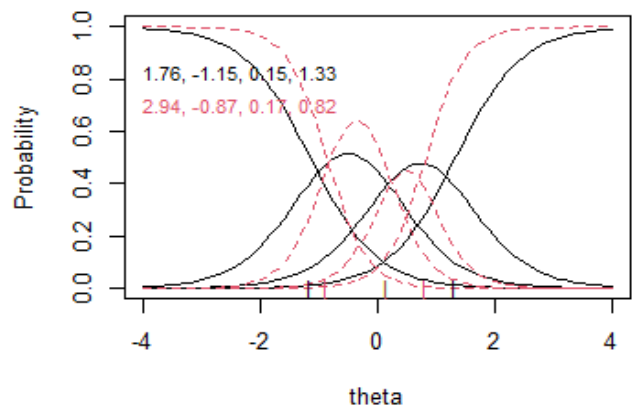

Differences in Item True Score Functions

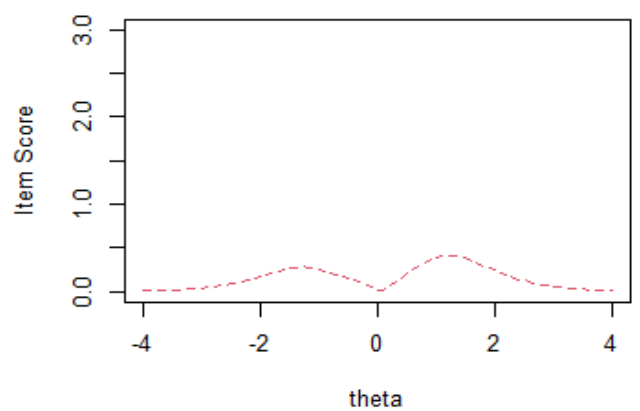

Impact (Weighted by Density)

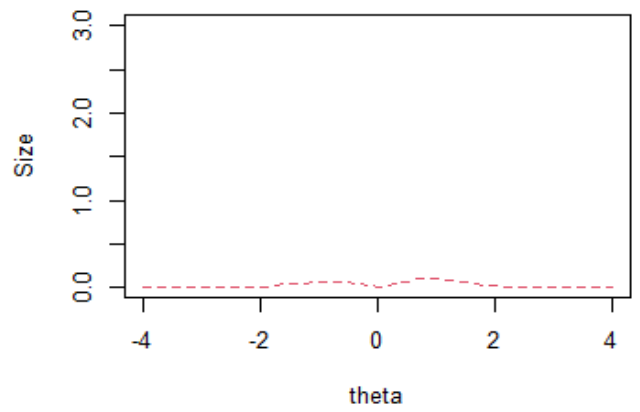




\section{Differential item functioning (DIF) - ethnicity}

As can be seen from Table 2, no items were flagged for DIF. Specifically, there were no significant difference tests at the $p$ $<0.01$ level between models and all changes in beta parameters were well below $5 \%$. Therefore, it was not possible to generate any meaningful plots to accompany the table.

Table 2. DIF test based on participants' ethnicity

\begin{tabular}{|c|c|c|c|c|c|c|c|}
\hline Item \# & M1 vs M2 & M1 vs M3 & M2 vs M3 & $\begin{array}{c}\mathbf{R}^{\mathbf{2}} \\
\text { (M1, M2) }\end{array}$ & $\begin{array}{c}\mathbf{R}^{\mathbf{2}} \\
\text { (M1, M3) }\end{array}$ & $\begin{array}{c}\mathbf{R}^{\mathbf{2}} \\
\text { (M2, } \mathbf{M 3})\end{array}$ & $\begin{array}{c}\Delta \boldsymbol{\beta} \\
\text { (M1, } \mathbf{M 2} \text { ) }\end{array}$ \\
\hline 1 & 0.244 & 0.438 & 0.622 & 0.003 & 0.005 & 0.001 & 0.011 \\
\hline 2 & 0.262 & 0.494 & 0.699 & 0.004 & 0.004 & 0.001 & 0.013 \\
\hline 3 & 0.533 & 0.611 & 0.489 & 0.002 & 0.003 & 0.002 & 0.002 \\
\hline 4 & 0.558 & 0.165 & 0.070 & 0.001 & 0.008 & 0.006 & 0.006 \\
\hline 5 & 0.371 & 0.557 & 0.600 & 0.003 & 0.004 & 0.001 & 0.003 \\
\hline 6 & 0.845 & 0.199 & 0.059 & 0.001 & 0.009 & 0.008 & 0.002 \\
\hline 7 & 0.723 & 0.572 & 0.322 & 0.001 & 0.004 & 0.003 & 0.001 \\
\hline 8 & 0.868 & 0.865 & 0.608 & 0.000 & 0.002 & 0.002 & 0.002 \\
\hline 9 & 0.181 & 0.133 & 0.162 & 0.011 & 0.022 & 0.012 & 0.018 \\
\hline
\end{tabular}

Notes. $M=$ model number chi-square test; statistical significance $<0.01 ; R^{2}=$ McFadden pseudo- $R^{2} ; \Delta \beta=$ change in beta for model 1 and model 2.

\section{Differential item functioning (DIF) - sample}

Table 3 shows that items 1, 3, 4, 8, and 9 were flagged for DIF. For items 1, 3, 4, and 8, the impacts were of negligible magnitude. Item 9's change in beta was $6.6 \%$ (i.e., above the $5 \%$ cut-off). However, given the intensity of the item, the item text itself, and the difference in sample sizes (327 versus 2254), the impact can be considered minor again.

Table 3. DIF test for the ODI based on participants' sample

\begin{tabular}{|c|c|c|c|c|c|c|c|}
\hline Item \# & M1 vs M2 & M1 vs M3 & M2 vs M3 & $\begin{array}{c}\mathbf{R}^{2} \\
\text { (M1, M2) }\end{array}$ & $\begin{array}{c}\mathbf{R}^{\mathbf{2}} \\
\text { (M1, M3) }\end{array}$ & $\begin{array}{c}\mathbf{R}^{\mathbf{2}} \\
\text { (M2, M3) }\end{array}$ & $\begin{array}{c}\Delta \boldsymbol{\beta} \\
\text { (M1, M2) }\end{array}$ \\
\hline 1 & 0.000 & 0.000 & 0.180 & 0.009 & 0.003 & 0.003 & 0.000 \\
\hline 2 & 0.917 & 0.937 & 0.729 & 0.000 & 0.000 & 0.000 & 0.000 \\
\hline 3 & 0.000 & 0.000 & 0.261 & 0.011 & 0.008 & 0.008 & 0.000 \\
\hline 4 & 0.000 & 0.000 & 0.882 & 0.007 & 0.005 & 0.005 & 0.000 \\
\hline 5 & 0.126 & 0.301 & 0.812 & 0.000 & 0.000 & 0.000 & 0.000 \\
\hline 6 & 0.058 & 0.051 & 0.126 & 0.002 & 0.001 & 0.001 & 0.000 \\
\hline 7 & 0.587 & 0.281 & 0.134 & 0.000 & 0.000 & 0.000 & 0.000 \\
\hline 8 & 0.001 & 0.001 & 0.113 & 0.003 & 0.002 & 0.002 & 0.000 \\
\hline 9 & 0.000 & 0.000 & 0.909 & 0.066 & 0.033 & 0.033 & 0.000 \\
\hline
\end{tabular}

Notes. $\mathrm{M}=$ model number chi-square test; statistical significance $<0.01 ; R^{2}=$ McFadden pseudo- $R^{2} ; \Delta \beta=$ change in beta for model 1 and model 2. 
Item True Score Functions - Item 1

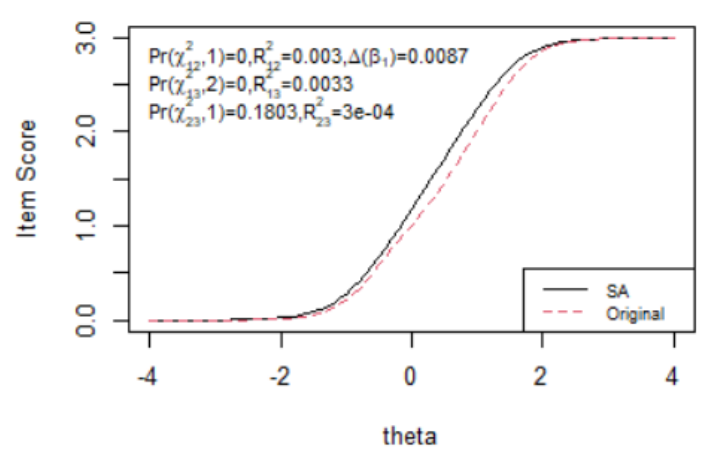

Item Response Functions

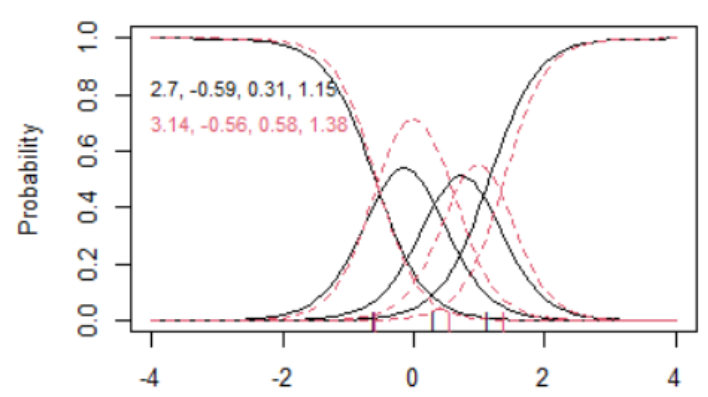

Item True Score Functions - Item 3

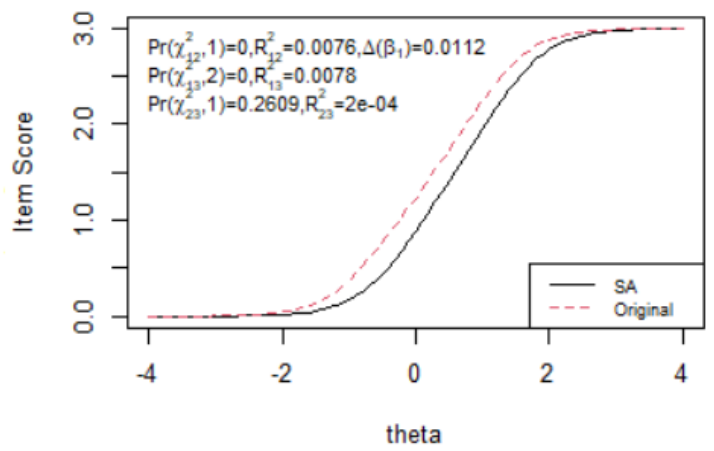

Item Response Functions

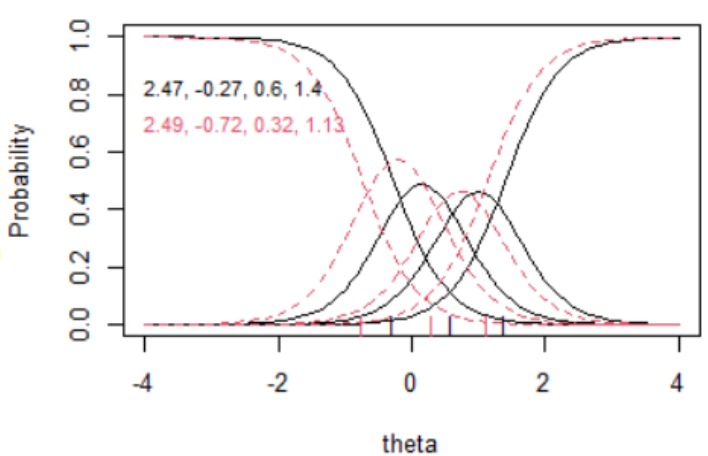

Differences in Item True Score Functions

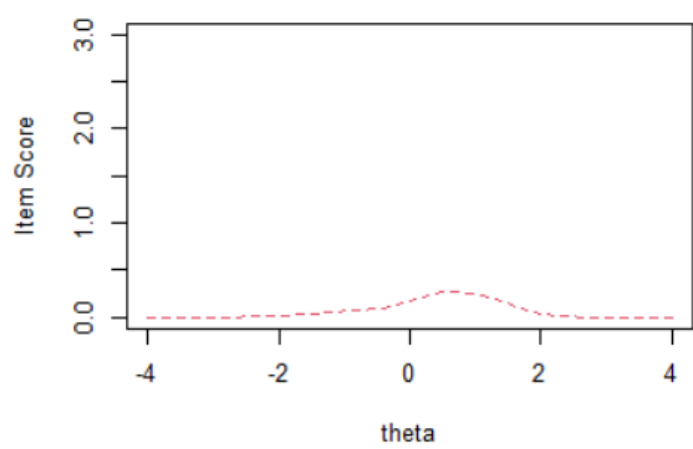

Impact (Weighted by Density)

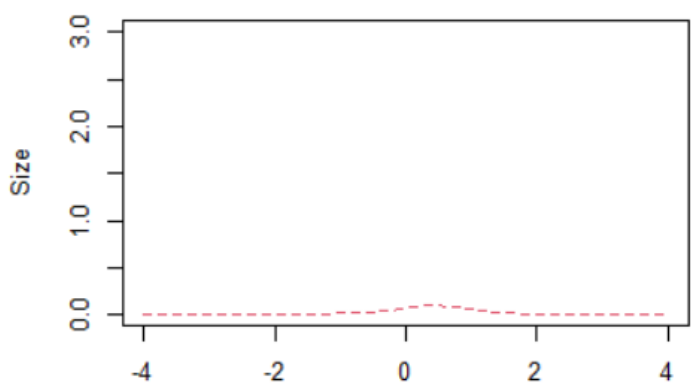

Differences in Item True Score Functions

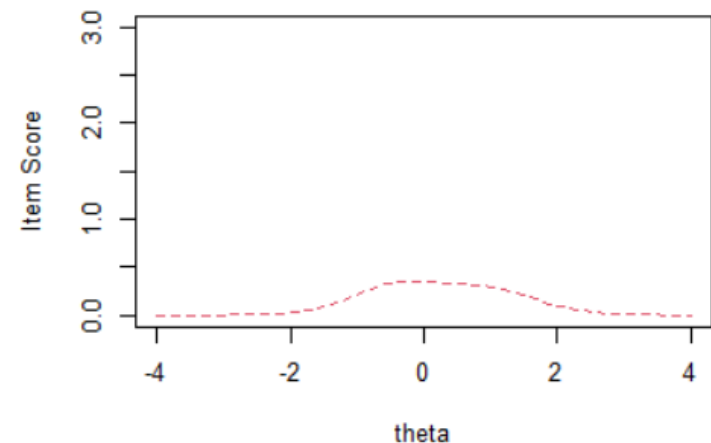

Impact (Weighted by Density)

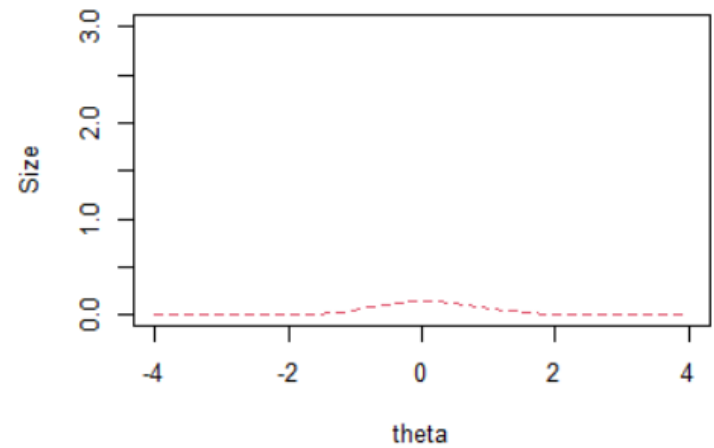


Item True Score Functions - Item 4

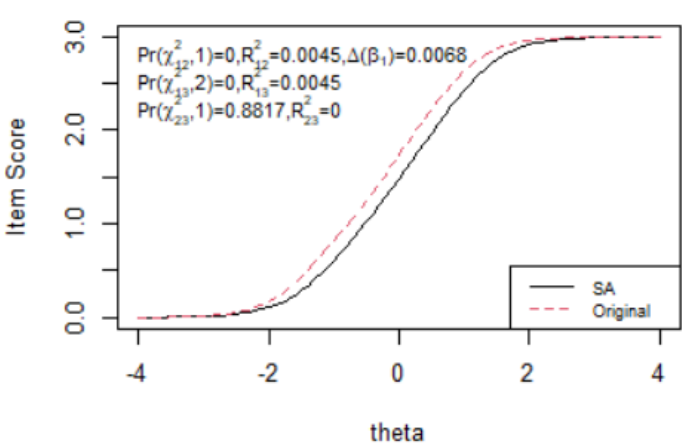

Item Response Functions

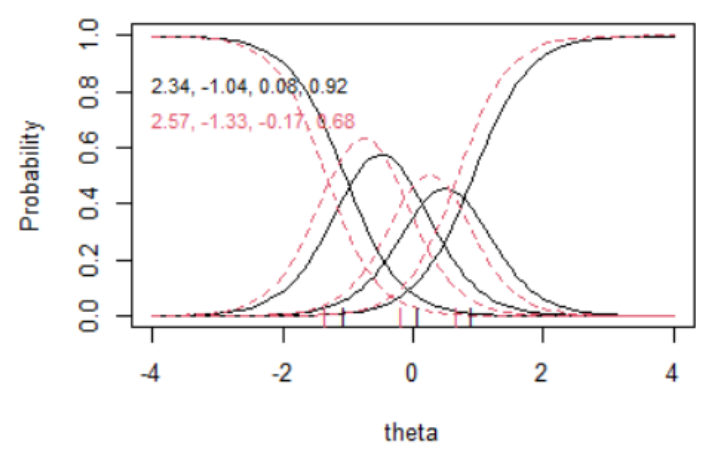

Item True Score Functions - Item 8

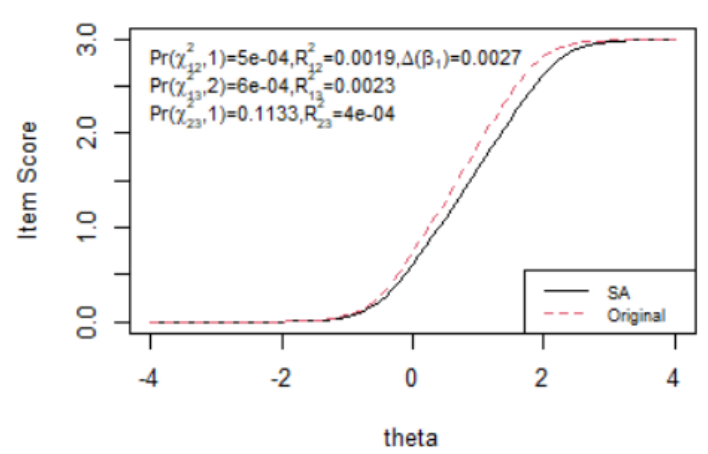

Item Response Functions

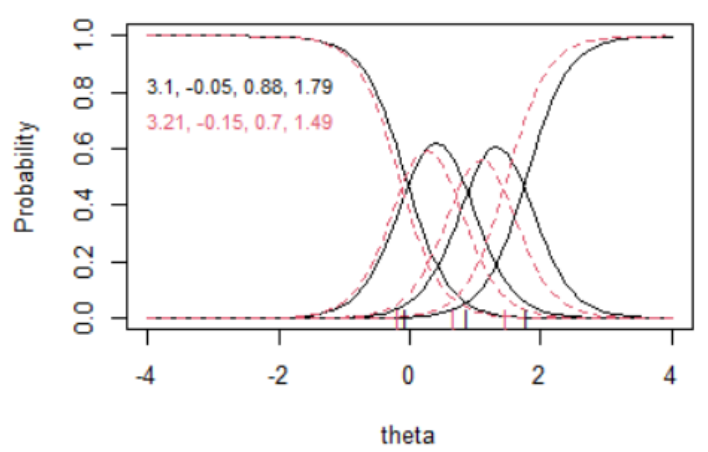

Differences in Item True Score Functions

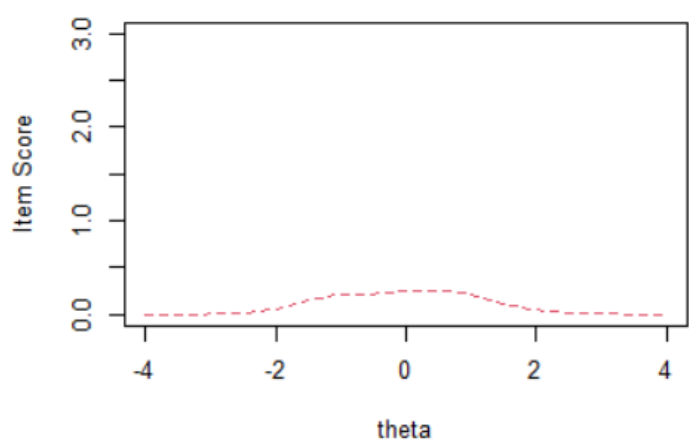

Impact (Weighted by Density)

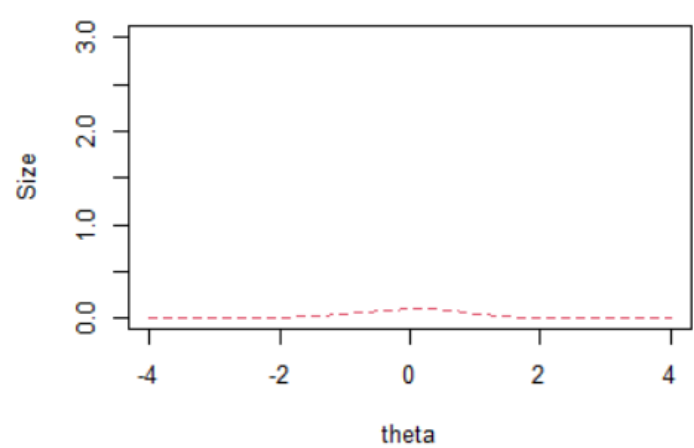

Differences in Item True Score Functions

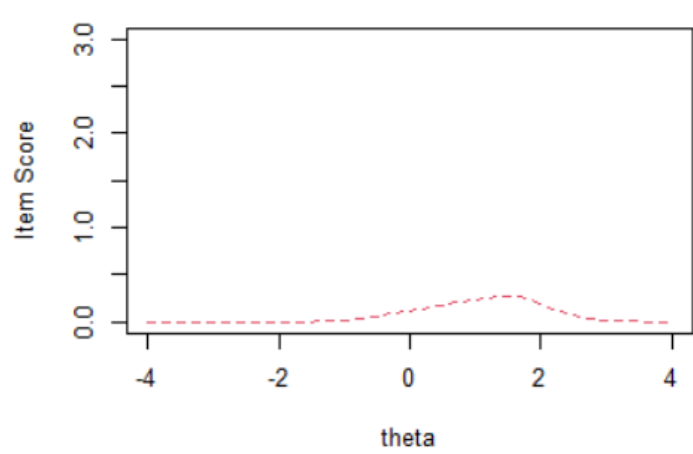

Impact (Weighted by Density)

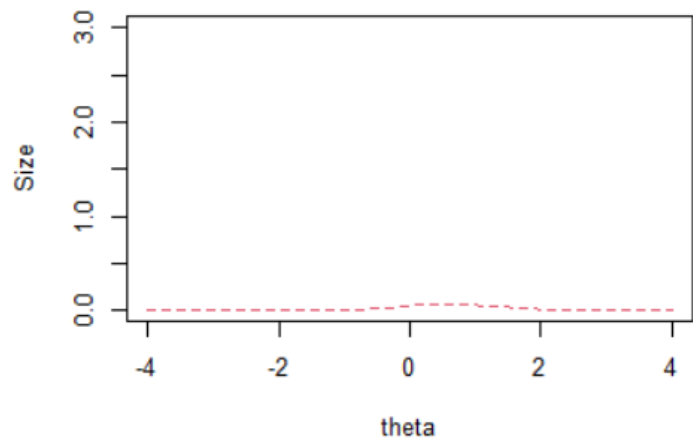


Item True Score Functions - Item 9

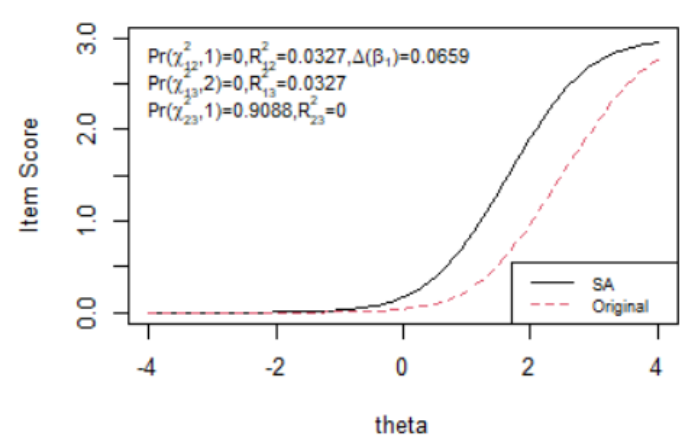

Item Response Functions

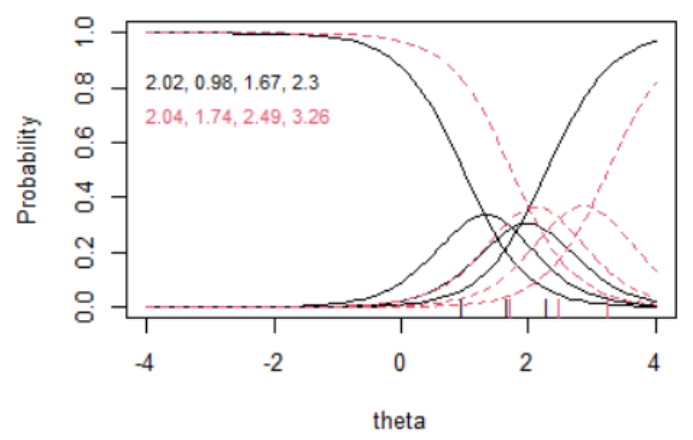

Differences in Item True Score Functions

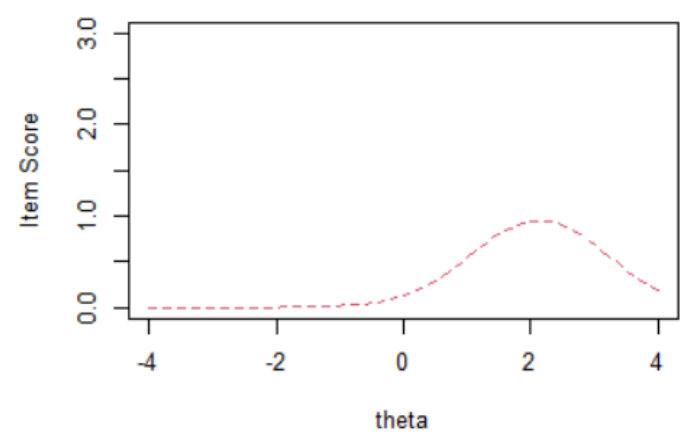

Impact (Weighted by Density)

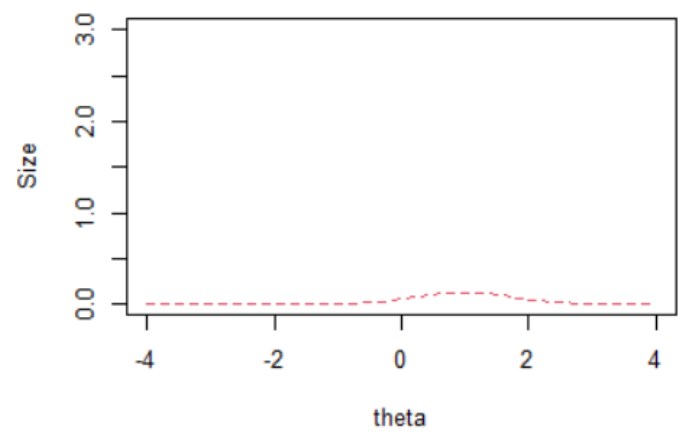

\section{Differential item functioning (DIF) - age group}

Only the first three age groups were viable for DIF testing due to group sizes. As can been seen from Table 4, no items were flagged for DIF. None of the model chi-square tests were significant and the changes in beta parameters were all well below $5 \%$.

Table 4. DIF test based on participants' age group

\begin{tabular}{|c|c|c|c|c|c|c|c|}
\hline Item \# & M1 vs M2 & M1 vs M3 & M2 vs M3 & $\begin{array}{c}\mathbf{R}^{\mathbf{2}} \\
\text { (M1, M2) }\end{array}$ & $\begin{array}{c}\mathbf{R}^{\mathbf{2}} \\
\text { (M1, M3) }\end{array}$ & $\begin{array}{c}\mathbf{R}^{\mathbf{2}} \\
\text { (M2, } \mathbf{M 3})\end{array}$ & $\begin{array}{c}\Delta \boldsymbol{\beta} \\
\text { (M1, M2) }\end{array}$ \\
\hline 1 & 0.131 & 0.183 & 0.341 & 0.010 & 0.005 & 0.008 & 0.003 \\
\hline 2 & 0.866 & 0.509 & 0.222 & 0.000 & 0.000 & 0.005 & 0.004 \\
\hline 3 & 0.488 & 0.491 & 0.372 & 0.006 & 0.002 & 0.005 & 0.003 \\
\hline 4 & 0.840 & 0.661 & 0.357 & 0.005 & 0.001 & 0.003 & 0.003 \\
\hline 5 & 0.648 & 0.875 & 0.840 & 0.001 & 0.001 & 0.002 & 0.001 \\
\hline 6 & 0.279 & 0.612 & 0.935 & 0.011 & 0.004 & 0.004 & 0.000 \\
\hline 7 & 0.422 & 0.152 & 0.083 & 0.005 & 0.003 & 0.010 & 0.008 \\
\hline 8 & 0.421 & 0.736 & 0.872 & 0.018 & 0.003 & 0.004 & 0.001 \\
\hline 9 & 0.599 & 0.185 & 0.075 & 0.018 & 0.004 & 0.022 & 0.018 \\
\hline
\end{tabular}

Notes. $\mathrm{M}=$ model number chi-square test; statistical significance $<0.01 ; R^{2}=M c F a d d e n$ pseudo- $R^{2} ; \Delta \beta=$ change in beta for model 1 and model 2. 